\title{
Chronic Kidney Disease as a Result of Secondary Oxalate Nephropathy From Excess Dietary Intake of Peanut Butter and Coffee
}

\author{
Ashkan Salamatipour ${ }^{\mathrm{a}, \mathrm{b}}$, d, Tina Moazezi ${ }^{\mathrm{b}}$, Suha Moten ${ }^{\mathrm{c}}$, \\ Laurie Anne Berga ${ }^{\mathrm{a}}$, Anis Abdul Rauf ${ }^{\mathrm{a}}$
}

\begin{abstract}
Excess production or absorption of oxalate can lead to hyperoxaluria and subsequent kidney injury. While many etiologies of hyperoxaluria have been well studied, diet-induced secondary oxalate nephropathy is a rare cause of renal damage that is still poorly understood. In this report, we present a 71-year-old man who lacked any known risk factors for secondary oxalate nephropathy other than a diet that was unusually high in peanut butter and coffee. The patient developed severe acute kidney injury, which resulted in end-stage renal disease and dependence on hemodialysis. Renal biopsy showed oxalate crystal buildup to be the culprit. It is likely that these cases of diet-induced secondary oxalate nephropathy have a strong genetic component that is yet to be fully understood. As the general public is likely unaware of the significance of dietary oxalate, we believe it is important to quickly identify and educate patients that have relevant risk factors to prevent development of acute or chronic kidney disease.
\end{abstract}

Keywords: Secondary oxalate nephropathy; Dietary hyperoxaluria

\section{Introduction}

Hyperoxaluria, or the excess excretion of oxalate through urine, has been known to cause nephrolithiasis and kidney damage. A significant majority of patients that develop kidney injury as a result of hyperoxaluria have a known history of either primary hyperoxalurias or secondary oxaluria, which

Manuscript submitted October 4, 2019, accepted October 16, 2019

aDepartment of Internal Medicine, AMITA Health Adventist Medical System, Bolingbrook, Glen Oaks, Hinsdale, La Grange, 911 N Elm St \#102, Hinsdale, IL 60521, USA

bMidwestern University, Chicago College of Osteopathic Medicine, Downers Grove, IL, USA

'Loyola University, Chicago, IL, USA

${ }^{\mathrm{d} C o r r e s p o n d i n g ~ A u t h o r: ~ A s h k a n ~ S a l a m a t i p o u r, ~ M i d w e s t e r n ~ U n i v e r s i t y, ~ C h i-~}$ cago College of Osteopathic Medicine, Downers Grove, IL, USA.

Email: asalamatipour84@midwestern.edu

doi: https://doi.org/10.14740/wjnu399 both lead to increased levels of urinary oxalate through various mechanisms. Primary hyperoxalurias, which can lead to excess production and deposition of oxalate in the kidneys, are due to genetic errors causing improper glyoxylate metabolism [1-3]. On the other hand, secondary hyperoxaluria is a consequence of increased oxalate absorption through the gastrointestinal tract, usually as a result of surgery (e.g. Roux-en-Y gastric bypass, jejunoileal bypass), fat malabsorption disorders (e.g. sprue, Crohn's disease), or medications that cause diarrhea (e.g. octreotide, orlistat, mycophenolate) [4-11]. Additionally, ethylene glycol (antifreeze) ingestion or high intake of vitamin $\mathrm{C}$, along with dietary deficiency of magnesium or calcium, has also been known to cause hyperoxaluria and oxalate crystal formation [12-14].

While the mechanisms behind these etiologies have been well studied, secondary oxalate nephropathy due to high dietary intake of oxalate-rich foods in the absence of the above etiologies is incredibly rare and poorly understood. Foods known to be highest in oxalate include spinach, nuts, chocolate and coffee, and it is possible that overconsumption of these foods may lead to oxalate deposition in the kidney and lead to chronic pathologic changes $[15,16]$. In this report, we present a 71-year-old man who came in with severe acute kidney injury (AKI) and was found to have secondary oxalate nephropathy in the absence of any causes other than a diet high in peanut butter and coffee.

\section{Case Report}

A 71-year-old Caucasian man came into the emergency department with severe shortness of breath and weakness of 2 weeks duration, which had culminated in audible wheezing and inability to support his own weight. In the emergency department, he was found to have severe AKI, with a sodium of 120 $\mathrm{mmol} / \mathrm{L}$, potassium of $5.6 \mathrm{mmol} / \mathrm{L}$, calcium of 7.5 , blood urea nitrogen of $126 \mathrm{mg} / \mathrm{dL}$, creatinine of $15.11 \mathrm{mg} / \mathrm{dL}$ and a glomerular filtration rate of $2.8 \mathrm{~mL} / \mathrm{min} / 1.73 \mathrm{~m}^{2}$. Noteworthy vital signs and physical exam findings included a blood pressure of $142 / 63 \mathrm{~mm} \mathrm{Hg}$, weight of $158 \mathrm{~kg}$ with a body mass index (BMI) of 40 and bilateral pretibial edema. Urinalysis revealed hematuria and minimal proteinuria. The 24-h urinary oxalate or calcium measurements were not available.

The patient had a personal medical history consisting of 


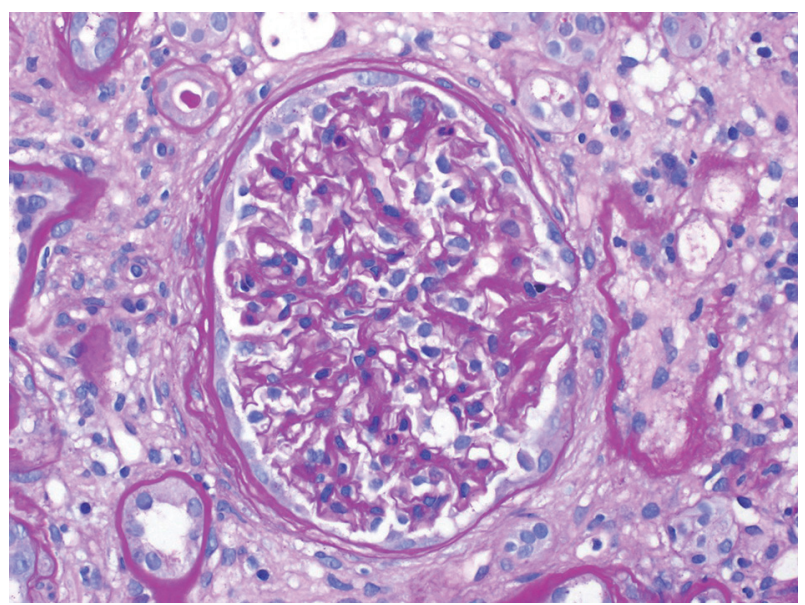

Figure 1. Loop wrinkling, periglomerular fibrosis.

type II diabetes mellitus treated with metformin, hyperlipidemia treated with atorvastatin, peripheral neuropathy treated with gabapentin, gout, and severe degenerative joint disease. The patient denied any family or personal history of renal disease, kidney stones, Crohn's disease, sprue, or any other gastrointestinal disorders. He also denied any abdominal surgeries, tobacco, alcohol, illicit drugs, ethylene glycol consumption, non-steroidal anti-inflammatory drugs, or exposure to contrast media. Upon admission to the hospital, the patient's previous medical records from 2 years prior showed him to have had a baseline serum creatinine of $1.6 \mathrm{mg} / \mathrm{dL}$, but with no known etiology or prior follow-up with a nephrologist.

After several days of care and an unremarkable renal ultrasound, a renal biopsy was ordered. The pathology demonstrated 13 out of 23 glomeruli to be globally sclerotic, as well as areas of capillary loop wrinkling and periglomerular fibrosis (Fig. 1). Patchy areas of acute tubular injury were present, with moderate numbers of injured tubules containing luminal calcium oxalate crystals, consistent with oxalate nephropathy (Figs. 2 and 3). Additionally, there was severe interstitial fibrosis and tubular atrophy involving an estimated $60 \%$ of the

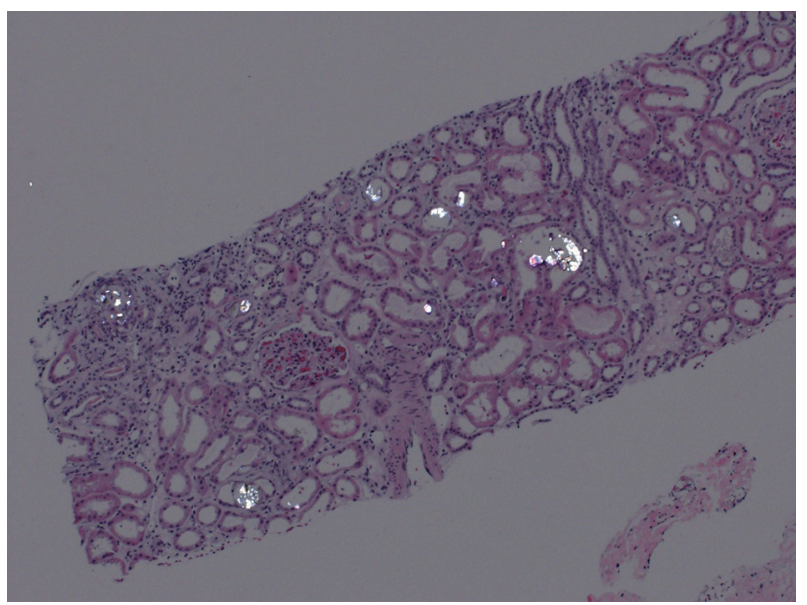

Figure 2. Polarized image showing calcium oxalate crystals in the tubules.

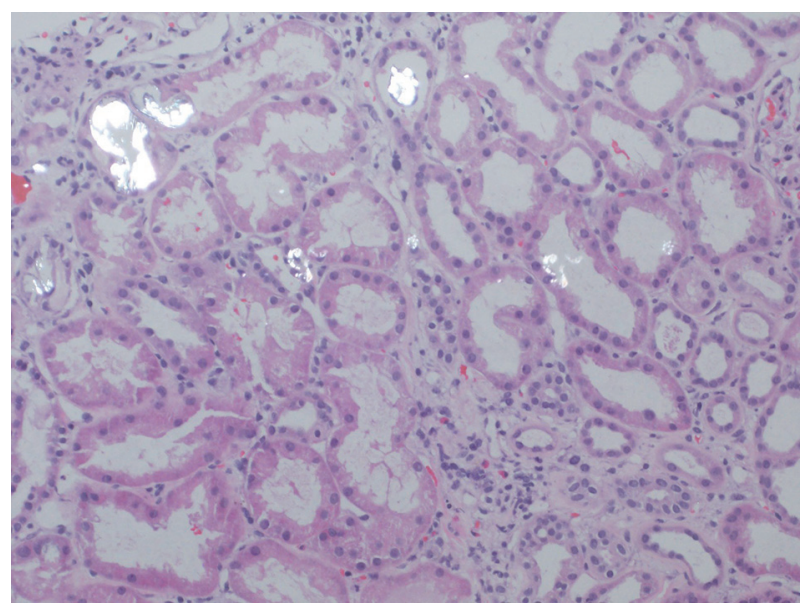

Figure 3. Injured tubules with luminal oxalate crystals.

submitted parenchyma, and moderate fibrointimal thickening of small arteries and severe hyalinosis of arterioles (Fig. 4). Immunofluorescence staining for IgG, IgM, IgA, C3, C1q, albumin and fibrinogen were negative, and kappa and lambda light chains stained equally throughout the tubulointerstitium.

Following the discovery of oxalate crystals in the patient's renal biopsy, he was questioned in detail on his diet and lifestyle habits. He said he was very conscious of his fluid intake and would consume large amounts of water, but admitted to a diet that was consistently heavy in nuts and coffee. He claimed that he would very frequently binge on peanut butter by eating up to half a jar in a single sitting, along with one handful of unsalted mixed nuts or peanuts 2 - 4 times weekly, and 3 - 4 large cups of coffee daily. Moreover, he claimed to consume moderate amounts of calcium by eating cheese and yogurt almost every day, but denied the consumption of vitamin $\mathrm{C}$ supplements. The patient was advised to limit his intake of oxalate-rich foods, and told to continue hemodialysis three times weekly with the hope that we could return his kidney function to a point of stability and independence. Three weeks after presenting to the hospital and starting dialysis, the patient's base-

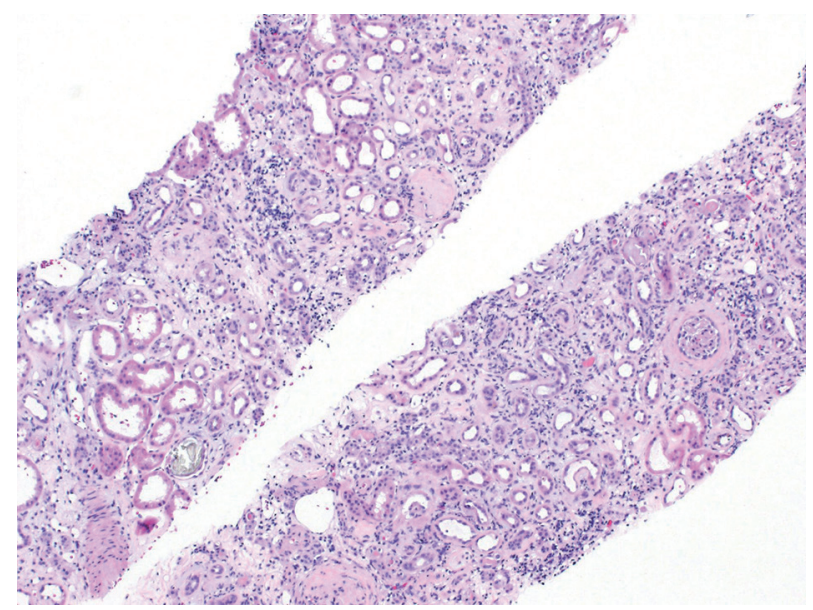

Figure 4. H\&E stain showing interstitial fibrosis and inflammation. H\&E: hematoxylin and eosin. 
line serum creatinine was $5.46 \mathrm{mg} / \mathrm{dL}$, his blood urea nitrogen was $38 \mathrm{mg} / \mathrm{dL}$ and his glomerular filtration rate was $11 \mathrm{~mL} /$ $\mathrm{min} / 1.73 \mathrm{~m}^{2}$, with a $24-\mathrm{h}$ urine output volume of $3 \mathrm{~L}$.

\section{Discussion}

Hyperoxaluria has been known to cause nephrolithiasis and inflammation in the kidney, leading to fibrosis and other chronic changes that can eventually lead to end-stage renal disease. However, in these cases, patients usually have identifiable issues that cause the excess production of oxalate (primary hyperoxalurias) or increased enteric uptake of oxalate due to gastrointestinal surgeries, malabsorption disorders, or diarrhea-inducing medications [4-11]. Nonetheless, our case is interesting because our patient did not have a single one of these known risk factors, and still developed secondary oxalate nephropathy as shown by renal biopsy. The only known contributing factor in this patient was his high daily intake of nuts, peanut butter and coffee, which are well-documented high-oxalate products $[15,16]$.

Although the patient did have diabetes mellitus for 6 years prior to his episode of AKI, it is unlikely that this was the cause of his renal injury since the peak incidence of diabetic nephropathy is $10-20$ years after a clinical diagnosis of diabetes [17]. Additionally, the patient's records demonstrated him to be compliant with all his medications and his HbAlc had been well controlled between $5.4 \%$ and $5.8 \%$ for the last several years. Thus, while any comorbid conditions may have led to slight progression of his renal interstitial fibrosis, both his past medical history and the biopsy results suggested his acute renal injury was a result of a hyperoxaluric state. Moreover, previous studies have shown that patients with a high BMI are more likely to develop calcium oxalate kidney stones, and since our patient had a BMI of 40, it is likely that his weight played a factor in oxalate deposition despite his negative history of nephrolithiasis $[18,19]$.

Lastly, this case is unique because of the patient's large daily consumption of water and calcium, and low consumption of vitamin $\mathrm{C}$, which have all been shown to be significant factors in calcium oxalate deposition $[13,14]$. In an attempt to find the cause of this patient's susceptibility to diet-induced secondary oxalate nephropathy, we came upon a study that showed mice with greater levels of the cryopyrin protein initiated a larger inflammatory response leading to greater renal injury when given an oxalate-rich diet [20]. At the same time, cryopyrin-null mice were immune to developing end-stage renal disease in response to high oxalate insult, showing that there is likely a significant genetic component to these cases of secondary oxalate nephropathy. More importantly, this and other similar studies have shown a mechanistic connection between oxalate nephropathy and crystal arthropathies such as gout, which may possibly be an underappreciated set of comorbidities, given that our patient and two other patients from similar case reports of secondary oxalate nephropathy all had gout [20-24].

Cases of diet-induced secondary oxalate nephropathy are rare and, to our knowledge, this is the first reported case of a patient who developed AKI after consuming large amounts of peanut butter and coffee. With the scarcity of information in the literature, it is unclear how to identify susceptible individuals, and how likely they are to recover from the fulminant renal insult caused by oxalate. While previous reports generally suggest that patients will need to remain on dialysis, our case may soon suggest otherwise $[13,25]$. Our patient's glomerular filtration rate went from $2.8 \mathrm{~mL} / \mathrm{min} / 1.73 \mathrm{~m}^{2}$ at the time of hospital admission to $11 \mathrm{~mL} / \mathrm{min} / 1.73 \mathrm{~m}^{2}$ just a few weeks later. Additionally, a study that examined the molecular mechanisms of oxalate nephropathy showed that the oxalate caused renal injury in two ways: it induced apoptosis of renal tubular cells, and it crystallized in the tubules causing obstruction [26]. Thus, if a patient receives dialysis soon enough, it may be possible to clear some of the tubular obstruction and allow time for the renal epithelial cells to regenerate, giving the patient a chance to wean off of dialysis once enough kidney function has been regained. Nonetheless, it is important to identify patients with risk factors for oxalate nephropathy and educate them on the importance of a low-oxalate/high-calcium diet, and encourage proper management of any other comorbidities such as diabetes and obesity.

\section{Acknowledgments}

None to declare.

\section{Financial Disclosure}

None to declare.

\section{Conflict of Interest}

All authors declare no conflict of interest.

\section{Informed Consent}

The patient has given their informed consent for the publication of this report.

\section{Author Contributions}

AS was responsible for the patient's care, data collection and writing the manuscript. TM was responsible for data collection, literature review and writing the manuscript. SM helped with literature review. LAB and AAR were responsible for the patient's care.

\section{References}

1. Hoppe B. An update on primary hyperoxaluria. Nat Rev Nephrol. 2012;8(8):467-475.

2. Cochat P. Primary hyperoxaluria type 1. Kidney Int. 
1999;55(6):2533-2547.

3. Bhasin B, Urekli HM, Atta MG. Primary and secondary hyperoxaluria: Understanding the enigma. World J Nephrol. 2015;4(2):235-244.

4. Nelson WK, Houghton SG, Milliner DS, Lieske JC, Sarr MG. Enteric hyperoxaluria, nephrolithiasis, and oxalate nephropathy: potentially serious and unappreciated complications of Roux-en-Y gastric bypass. Surg Obes Relat Dis. 2005;1(5):481-485.

5. Hassan I, Juncos LA, Milliner DS, Sarmiento JM, Sarr MG. Chronic renal failure secondary to oxalate nephropathy: a preventable complication after jejunoileal bypass. Mayo Clin Proc. 2001;76(7):758-760.

6. Hueppelshaeuser R, von Unruh GE, Habbig S, Beck BB, Buderus S, Hesse A, Hoppe B. Enteric hyperoxaluria, recurrent urolithiasis, and systemic oxalosis in patients with Crohn's disease. Pediatr Nephrol. 2012;27(7):1103-1109.

7. McDonald GB, Earnest DL, Admirand WH. Hyperoxaluria correlates with fat malabsorption in patients with sprue. Gut. 1977;18(7):561-566.

8. Parasuraman R, Venkat KK. Crystal-induced kidney disease in 2 kidney transplant recipients. Am J Kidney Dis. 2010;55(1):192-197.

9. Singh A, Sarkar SR, Gaber LW, Perazella MA. Acute oxalate nephropathy associated with orlistat, a gastrointestinal lipase inhibitor. Am J Kidney Dis. 2007;49(1):153157.

10. Gariani K, de Seigneux S, Courbebaisse M, Levy M, Moll S, Martin PY. Oxalate nephropathy induced by octreotide treatment for acromegaly: a case report. J Med Case Rep. 2012;6:215.

11. Hoppe B, von Unruh GE, Blank G, Rietschel E, Sidhu $\mathrm{H}$, Laube N, Hesse A. Absorptive hyperoxaluria leads to an increased risk for urolithiasis or nephrocalcinosis in cystic fibrosis. Am J Kidney Dis. 2005;46(3):440-445.

12. Lamarche J, Nair R, Peguero A, Courville C. Vitamin C-induced oxalate nephropathy. Int J Nephrol. 2011;2011:146927.

13. Hoppe B, Leumann E. Diagnostic and therapeutic strategies in hyperoxaluria: a plea for early intervention. Nephrol Dial Transplant. 2004;19(1):39-42.
14. Brogren M, Savage GP. Bioavailability of soluble oxalate from spinach eaten with and without milk products. Asia Pac J Clin Nutr. 2003;12(2):219-224.

15. Noonan SC, Savage GP. Oxalate content of foods and its effect on humans. Asia Pac J Clin Nutr. 1999;8(1):64-74.

16. Attalla K, De S, Monga M. Oxalate content of food: a tangled web. Urology. 2014;84(3):555-560.

17. Shahbazian H, Rezaii I. Diabetic kidney disease; review of the current knowledge. J Renal Inj Prev. 2013;2(2):7380 .

18. Siener R, Glatz S, Nicolay C, Hesse A. The role of overweight and obesity in calcium oxalate stone formation. Obes Res. 2004;12(1):106-113.

19. Trinchieri A, Croppi E, Montanari E. Obesity and urolithiasis: evidence of regional influences. Urolithiasis. 2017;45(3):271-278.

20. Knauf F, Asplin JR, Granja I, Schmidt IM, Moeckel GW, David RJ, Flavell RA, et al. NALP3-mediated inflammation is a principal cause of progressive renal failure in oxalate nephropathy. Kidney Int. 2013;84(5):895-901.

21. Mulay SR, Kulkarni OP, Rupanagudi KV, Migliorini A, Darisipudi MN, Vilaysane A, Muruve D, et al. Calcium oxalate crystals induce renal inflammation by NLRP3-mediated IL-1beta secretion. J Clin Invest. 2013;123(1):236246.

22. Martinon F, Petrilli V, Mayor A, Tardivel A, Tschopp J. Gout-associated uric acid crystals activate the NALP3 inflammasome. Nature. 2006;440(7081):237-241.

23. Humayun Y, Ball KC, Lewin JR, Lerant AA, Fulop T. Acute oxalate nephropathy associated with orlistat. J Nephropathol. 2016;5(2):79-83.

24. Khneizer G, Al-Taee A, Mallick MS, Bastani B. Chronic dietary oxalate nephropathy after intensive dietary weight loss regimen. J Nephropathol. 2017;6(3):126-129.

25. Niticharoenpong $\mathrm{K}$, Chalermsanyakorn $\mathrm{P}$, Panvichian R, Kitiyakara C. Acute deterioration of renal function induced by star fruit ingestion in a patient with chronic kidney disease. J Nephrol. 2006;19(5):682-686.

26. Fang HC, Lee PT, Lu PJ, Chen CL, Chang TY, Hsu CY, Chung HM, et al. Mechanisms of star fruit-induced acute renal failure. Food Chem Toxicol. 2008;46(5):1744-1752. 